\section{SAT0135 MAINTAINING REMISSION IN PATIENTS WITH ESTABLISHED RHEUMATOID ARTHRITIS WHILE TAPERING ETANERCEPT: AN INSIGHT IN THE TAPERA TRIAL}

Delphine Bertrand ${ }^{1}$, Julie Mannaerts ${ }^{1}$, Valérie Badot ${ }^{2}$, Luk Corluy ${ }^{3}$, Diederik De Cock ${ }^{1}$, Kathleen De Knop ${ }^{4}$, Isabelle de Wergifosse ${ }^{5}$, Elke Geens ${ }^{6}$, Ilse Hoffman ${ }^{4}$, Johan Joly ${ }^{5}$, Christine Langenaken ${ }^{3}$, Jan Lenaerts ${ }^{3}$, Joris Lenaerts ${ }^{5}$ Sofia Pazmino ${ }^{1}$, Veerle Stouten ${ }^{1}$, Veerle Taelman ${ }^{7}$, Mark Walschot ${ }^{5}$, Rene Westhovens ${ }^{1,5}$, Patrick Verschueren ${ }^{1,5} .{ }^{1} \mathrm{KU}$ Leuven, Skeletal Biology and Engineering Research Center, Leuven, Belgium; ${ }^{2}$ ULB Hôpital Erasme, Brussel, Belgium; ${ }^{3}$ Reuma Instituut, Hasselt, Belgium; ${ }^{4}$ GZA Sint-Augustinus, Antwerpen, Belgium; ${ }^{5}$ UZ Leuven, Rheumatology, Leuven, Belgium; ${ }^{6}$ ZNA Jan Palfijn, Antwerpen, Belgium; ${ }^{7}$ Heilig Hart Ziekenhuis, Leuven, Belgium

Background: EULAR 2016 recommendations for the management of Rheumatoid Arthritis (RA) suggest to consider tapering of biological Disease-Modifying Antirheumatic Drugs (bDMARDs) in patients in sustained remission. More insight on the effect of tapering strategies is needed in daily practice.

Objectives: To investigate maintaining disease control after spacing dosages of etanercept $50 \mathrm{mg}$ from weekly to every other week (EOW) in a pragmatic randomized controlled trial (RCT).

Methods: Patients with RA who were in remission according to the disease activity score 28 (DAS28) remission criteria for at least 6 months and treated with etanercept $50 \mathrm{mg}$ weekly for at least a year were included in the one-year non-blinded multicentre TapERA (Tapering Etanercept in RA) RCT (EudraCTnumber 2012-004631-22). Patients were 1:1 randomly assigned to continue etanercept $50 \mathrm{mg}$ weekly or to taper to $50 \mathrm{mg}$ EOW. Patients who lost remission (DAS28 C-Reactive Protein $(C R P) \geq 2.6)$ received nonsteroidal anti-inflammatory drugs and if needed were re-escalated to etanercept weekly. If remission was still not reached, treatment had to be adapted, at the discretion of the treating rheumatologist. The outcomes were proportion of patients in DAS28 remission at 6 and 12 months, proportion in sustained remission and proportion regaining remission after reintroducing weekly etanercept (intention to treat analysis). Missing components of DAS28 were imputed using expectation maximization.

Results: In total, 69 patients $(69.6 \%$ female $)$ with a mean \pm standard deviation (SD) age of $55.7 \pm 11.3$ years and a mean \pm SD disease duration of $14.7 \pm 8.0$ years were included. The weekly and EOW group consisted of 35 and 34 patients respectively. At the month 6 visit $77.1 \%$ patients of the weekly and $76.5 \%$ of the EOW group were in remission $(p=0.947)$ and $68.6 \%$ and $58.8 \%$ respectively maintained remission on every visit $(p=0.400)$ (Table 1$)$

Three $(8.6 \%)$ patients of the weekly and $11(32.4 \%)$ of the EOW group required a treatment adaptation, which was statistically significantly different $(p=0.014)$. There was no difference between the weekly and EOW group in the mean \pm SD DAS28 CRP at the first time of losing remission in patients flaring, namely $3.1 \pm 0.6$ and $3.1 \pm 0.5$ respectively $(\mathrm{p}=0.957)$.

Eleven patients were re-escalated to a weekly treatment after a mean \pm SD duration of $4.5 \pm 3.1$ months. Of these, $54.5 \%(6 / 11)$ regained remission after a mean \pm SD duration of $4.7 \pm 1.7$ months. Of the 5 patients not regaining remission, 1 switched to a weekly regimen on the last trial visit, 1 required switching to another bDMARD and 3 patients had no additional treatment changes (mean \pm SD DAS28 CRP at month 12: 3.4 \pm 0.2 ). Two patients of the EOW group restarted weekly etanercept while still being in remission.

\begin{tabular}{|c|c|c|c|}
\hline & Weekly group ( $n=35$ ) & EOW group ( $n=34)$ & p-value \\
\hline Remission at $\mathrm{M} 6 \mathrm{n}(\%)$ & $27(77.1)$ & $26(76.5)$ & 0.947 \\
\hline LDA at M6 $\mathrm{n}(\%)$ & $29(82.9)$ & $30(88.2)$ & 0.526 \\
\hline Sustained remission M6 $\mathrm{n}(\%)$ & $24(68.6)$ & $20(58.8)$ & 0.400 \\
\hline Sustained LDA M6 $\mathrm{n}(\%)$ & $29(82.9)$ & $26(76.5)$ & 0.510 \\
\hline Remission at M12 $\mathrm{n}(\%)$ & $30(85.7)$ & $24(70.6)$ & 0.128 \\
\hline LDA at $M 12 n(\%)$ & $33(94.3)$ & $28(82.4)$ & 0.122 \\
\hline Sustained remission M12 $\mathrm{n}(\%)$ & $21(60.0)$ & $15(44.1)$ & 0.187 \\
\hline Sustained LDA M12n(\%) & $25(71.4)$ & $24(70.6)$ & 0.939 \\
\hline $\begin{array}{l}\text { Patients with treatment adaptation } \\
\text { due to efficacy loss } n(\%)\end{array}$ & $3(8.6)$ & $11(32.4)$ & 0.014 \\
\hline
\end{tabular}

escalation to weekly respectively; EOW every other week, M month, LDA low disease activity (DAS28 CRP $\leq 3.2$ )

Conclusion: Approximately half of the EOW group was in sustained remission after 1 year and two thirds remained on their reassigned treatment. Furthermore, one in two patients were able to regain remission after reintroducing etanercept weekly. Therefore tapering of etanercept to EOW seems a feasible strategy in patients with RA in sustained remission, potentially leading to a beneficial cost-saving and safety profile.
Disclosure of Interests: Delphine Bertrand: None declared, Julie Mannaerts: None declared, Valérie Badot: None declared, Luk Corluy: None declared, Diederik De Cock: None declared, Kathleen De Knop: None declared, Isabelle de Wergifosse: None declared, Elke Geens: None declared, Ilse Hoffman: None declared, Johan Joly: None declared, Christine Langenaken: None declared, Jan Lenaerts: None declared, Joris Lenaerts: None declared, Sofia Pazmino: None declared, Veerle Stouten: None declared, Veerle Taelman: None declared, Mark Walschot: None declared, Rene Westhovens Grant/research support from: Bristol-Myers Squibb, Consultant for: Celltrion, Galapagos-Gilead, Patrick Verschueren Grant/research support from: Unrestricted Pfizer Grant for Early RA research

DOI: 10.1136/annrheumdis-2019-eular.3055

\section{SAT0136 CONSISTENCY WITH INTERNATIONAL GUIDELINES REGARDING MOTHERS WITH RHEUMATOLOGIC DISEASES EXPOSED TO TUMOUR NECROSIS FACTOR INHIBITORS (TNFI) DURING THE ANTE- AND POSTNATAL PERIODS AND CHANGE OVER TIME}

Stephanie Bond ${ }^{1,2}$, Laurel Young ${ }^{1}$, Katherine Poulsen ${ }^{1}$, Helen L. Barrett ${ }^{3}$, Andrew L. Taylor ${ }^{4}$, Claire Barrett ${ }^{1} .1$ University of Queensland, Brisbane, Australia; ' James Cook University, Townsville, Australia; ${ }^{3}$ Mater Health Services, South Brisbane, Australia; ${ }^{4}$ Royal Perth Hospital, Perth, Australia

Background: Many women with rheumatologic diseases require ongoing treatment with TNFi during pregnancy to maintain remission or low disease activity. Until recently, there has been a paucity of published evidence regarding the safe use of these medications during the ante- and postnatal periods to guide clinical practice.

Objectives: To observe compliance with current guidelines for TNFi therapy in Australian women with rheumatologic diseases during the anteand postnatal periods and change over time.

Methods: All Australian women with rheumatologic diseases, exposed to biologics (bDMARDs) and targeted synthetic disease modifying antirheumatic drugs (tsDMARDs) during the preconception, antenatal and/or postpartum periods were eligible to participate in the Pregnancy Exposed to Biological (PEB) study. Commencing in 2015, recruitment was via invitation from patient's treating rheumatologists, community groups, and social media. Following self-referral to the study, retrospective data was collected, including rheumatologic condition and management recommendations made by health professionals.

Results: Preliminary data is available for 37 infants born to 29 mothers from Feb 2009-Dec 2018. Of these, 32 women received TNFi. We assessed 3 outcomes - TNFi continuation during pregnancy, TNFi continuation during lactation and vaccination practice in infants exposed to TNFi. Women exposed to non-TNFi bDMARDs and tsDMARDs were excluded from this abstract, as these are not recommended for use during the perinatal period (2)

Specialist Society Guidelines from the British Society of Rheumatology and the British Health Professional in Rheumatology were published in 2016 regarding the safety of TNFi during pregnancy (2). Prior to guideline publication, $67 \%(n=16 / 24)$ of the women in PEB ceased their TNFi pre-conception. Of those in PEB who became pregnant subsequent to guideline publication, only $25 \%(n=2 / 8)$ ceased TNFi pre-conception. Overall, $40.6 \% \quad(n=13 / 32)$ women received a TNFi during the antenatal period consistent with guidelines, which improved from $33.3 \%$ ( $n=8 / 24)$ pre-publication, to $62.5 \%(n=5 / 8)$ post publication.

84.4\% $(\mathrm{n}=27 / 32)$ women exposed to TNFi breastfed their infants. Prior to availability of evidence regarding the safety of TNFi during lactation, $79.2 \%(n=19 / 24)$ of infants were breastfed. After publication, $100 \%(n=8 /$ 8) infants exposed to TNFi were breastfed.

In total, $96.9 \% \quad(n=31 / 32)$ of the infants exposed to TNFi in PEB were vaccinated. Rotavirus vaccine should have been delayed in $43.8 \% \quad(n=14 /$ 32) infants, but was not. $9.4 \%(n=3 / 32)$ infants had live vaccines deferred until only 3 months; $3.1 \% \quad(n=1 / 32)$ infants had live vaccines unnecessarily delayed. Only $3.1 \%(n=1 / 32)$ infants had live vaccines appropriately delayed until 7 months. Compliance with vaccination recommendations increased from $43.5 \% \quad(n=10 / 24)$ pre-publication to $62.5 \% \quad(n=5 / 8)$ post publication of guidelines.

Conclusion: Preliminary data from PEB suggests that there has been a shift in practice following the publication of Specialist Society Guidelines, with increasing numbers of women continuing TNFi therapy during pregnancy and the postpartum period, in keeping with current evidence. Compliance with vaccination recommendations could be improved to be more consistent with published international guidelines. 